\title{
A GAUSSIAN SMALL DEVIATION INEQUALITY FOR CONVEX FUNCTIONS
}

\author{
By Grigoris PAOURIS ${ }^{1}$ AND Petros VAlettas ${ }^{2}$

\section{Texas A\&M University and University of Missouri}

Let $Z$ be an $n$-dimensional Gaussian vector and let $f: \mathbb{R}^{n} \rightarrow \mathbb{R}$ be a convex function. We prove that

$$
\mathbb{P}(f(Z) \leq \mathbb{E} f(Z)-t \sqrt{\operatorname{Var} f(Z)}) \leq \exp \left(-c t^{2}\right),
$$

for all $t>1$ where $c>0$ is an absolute constant. As an application we derive variance-sensitive small ball probabilities for Gaussian processes.

1. Introduction. The purpose of this note is to establish a sharp distributional inequality for convex functions on Gauss' space $\left(\mathbb{R}^{n},\|\cdot\|_{2}, \gamma_{n}\right)$. Our goal and motivation stems from the attempt to strengthen the classical Gaussian concentration for special cases that are of interest in high-dimensional geometry. The Gaussian concentration phenomenon (see [1] and [20]) states that for any L-Lipschitz map $f: \mathbb{R}^{n} \rightarrow \mathbb{R}$ one has

$$
\mathbb{P}(|f(Z)-M|>t) \leq \exp \left(-\frac{1}{2} t^{2} / L^{2}\right),
$$

for all $t>0$, where $Z$ is $n$-dimensional standard Gaussian random vector and $M$ is a median for $f(Z)$. The above inequality follows from the solution to the isoperimetric problem in Gauss' space, which was proved independently by Borell in [2] and Sudakov and Tsirel'son in [33] and can be described by the following inequality:

$$
\gamma_{n}\left(A+t B_{2}^{n}\right) \geq \Phi\left(\Phi^{-1}\left(\gamma_{n}(A)\right)+t\right), \quad t>0,
$$

for all Borel sets $A \subseteq \mathbb{R}^{n}$, where $\Phi$ is the cumulative distribution function of a Gaussian random variable. Applying (1.2) for $A=\{f \leq M\}$, where $M$ is a median of $f$ and by taking into account that $A+t B_{2}^{n} \subseteq\{f \leq M+t L\}$ we obtain

$$
\gamma_{n}(f \leq M+t L) \geq \Phi(t) \quad \Longrightarrow \quad \gamma_{n}(f>M+t L) \leq 1-\Phi(t) .
$$

Finally, standard estimates for the function $\Phi$, such as (2.3), yield the result (we work similarly for the deviation below the median). In turn, this implies bounds on

Received November 2016; revised May 2017.

${ }^{1}$ Supported by NSF CAREER-1151711 Grant.

${ }^{2}$ Supported by NSF Grant DMS-1612936.

MSC2010 subject classifications. Primary 60D05; secondary 52A21, 52A23.

Key words and phrases. Ehrhard's inequality, concentration for convex functions, small ball probability, Johnson-Lindenstrauss lemma. 
the variance $\operatorname{Var} f(Z)$ for any Lipschitz map $f$ in terms of the Lipschitz constant $L$ (alternatively we may employ the Gaussian Poincaré inequality [6]):

$$
\operatorname{Var}[f(Z)] \leq L^{2} \text {. }
$$

The above inequalities are sharp for linear functionals. However, one can easily construct examples of convex functions (see Section 2) for which the above estimates are far from being optimal. On the other hand, the observation in [21], Corollary 3.2, that for $f$ being a norm, one has the stochastic dominance $\mathbb{P}(f(Z) \geq t) \geq \mathbb{P}(|\ell(Z)| \geq t)$ for all norm one linear functionals $\ell$, implies that (1.1) is sharp (up to absolute constants) in the large deviation regime $t>M$ (see also [22] and [30], Proposition 2.9, for details). Therefore, in this note the focus is on the one-sided small deviation inequality:

$$
\mathbb{P}(f(Z)-M<-t) \leq \frac{1}{2} \exp \left(-\frac{1}{2} t^{2} / L^{2}\right),
$$

which holds for all $t>0$ and for any $L$-Lipschitz map $f$. This inequality is of great importance in asymptotic geometric analysis, hence one would be interested in refined forms of (1.4). For different ranges of $t$, one can replace the Lipschitz constant \|\|$\nabla f\left\|_{2}\right\|_{L_{\infty}}$ by appropriately chosen moments of $\|\nabla f\|_{2}$. This is based on various Gaussian functional inequalities such as the logarithmic Sobolev inequalities, the Poincaré inequalities, the $(p, q)$-Poincaré inequalities and more (see [4, 20]). Even in that case, there exist examples of convex functions [e.g., $\left.f(x)=\max _{i \leq n}\left|x_{i}\right|\right]$ for which the $L_{2}$ norm of the gradient is much larger than the variance, therefore, these inequalities fail to capture the right order of concentration (see [5] for a detailed discussion of this phenomenon). Ideally, one would like to replace the Lipschitz constant in (1.4) by a statistical measure of dispersion, for example, the variance. Indeed this is the case for convex functions. Our main result reads as follows: For any convex map $f \in L_{2}\left(\gamma_{n}\right)$, one has

$$
\mathbb{P}(f(Z)-M<-t) \leq \frac{1}{2} \exp \left(-\frac{\pi}{1024} t^{2} / \operatorname{Var}[f(Z)]\right),
$$

for all $t>0$. In view of (1.3), this obviously improves the one-sided concentration inequality in the small deviation regime. We want to emphasize that the above inequality, unlike to most concentration inequalities which are isoperimetric in nature, does not follow by the Gaussian isoperimetry. Instead it is obtained by the convexity properties of the Gaussian measure, thus it could be viewed as a "new type" of concentration. The last but not least is that the function is not required to be Lipschitz in (1.5); instead it is valid for any convex function $f \in L_{2}\left(\gamma_{n}\right)$ [in fact, we may even prove a similar inequality to (1.5) by assuming weaker integrability condition for $f$; see Remark 2.4.1].

The rest of the paper is organized as follows: In Section 2, we present a proof of the main result. The key ingredient in our argument is Ehrhard's inequality [8], inspired by the approach of Kwapien in [17]. We conclude in Section 3 with some applications. 
2. Proof of the main result. Let $\Phi$ be the cumulative distribution function of a standard Gaussian random variable, that is,

$$
\Phi(x)=\frac{1}{\sqrt{2 \pi}} \int_{-\infty}^{x} e^{-z^{2} / 2} d z, \quad x \in \mathbb{R} .
$$

Ehrhard's inequality [8] states that for any two convex sets $A, B$ on $\mathbb{R}^{n}$ and for any $0<\lambda<1$ one has

$$
\Phi^{-1}\left[\gamma_{n}((1-\lambda) A+\lambda B)\right] \geq(1-\lambda) \Phi^{-1}\left[\gamma_{n}(A)\right]+\lambda \Phi^{-1}\left[\gamma_{n}(B)\right]
$$

Ehrhard's result was extended by Latała in [18] to the case that one of the two sets is Borel and the other is convex. Finally, in [3], Borell proved that it holds for all pairs of Borel sets. Recently, many different proofs of this fundamental inequality have appeared in the literature; see, for example, [12, 26, 34] and the references therein.

Our goal is to prove the following.

THEOREM 2.1. Let $Z$ be an n-dimensional standard Gaussian vector. Let $f$ be a convex function on $\mathbb{R}^{n}$ with $f \in L_{1}\left(\gamma_{n}\right)$ and let $M$ be a median for $f(Z)$. Then we have

$$
\mathbb{P}\left(f(Z)-M<-t \mathbb{E}(f(Z)-M)_{+}\right) \leq \Phi\left(-\frac{\sqrt{2 \pi}}{32} t\right),
$$

for all $t>0$.

Proof. Since $M$ is a median, we have $\mathbb{P}(f(Z) \leq M) \geq 1 / 2$. We may assume without loss of generality that $\mathbb{P}(f(Z) \leq M)=1 / 2$. Otherwise we have $\mathbb{P}(f=$ $M)>0$, and since $f$ is convex, we get $f \geq M$, thus the conclusion is trivially true. Note that the convexity of $f$ implies that the sub-level sets $\{f \leq t\}, t \in \mathbb{R}$ are convex and the function $F(t):=\mathbb{P}(f(Z) \leq t)$ is log-concave. The latter follows by the following inclusion:

$$
(1-\lambda)\{f \leq t\}+\lambda\{f \leq s\} \subseteq\{f \leq(1-\lambda) t+\lambda s\},
$$

for $t, s \in \mathbb{R}$ and $0 \leq \lambda \leq 1$ and the fact that $\gamma_{n}$ is log-concave measure (see [1], Section 1.8, for the related definition). Now, we may use Ehrhard's inequality from [8] (see also [1], Theorem 4.2.1) to get that the map $s \mapsto \Phi^{-1} \circ F(s), s \in \mathbb{R}$ is concave (for a proof see [1], Theorem 4.4.1). Therefore, we obtain

$$
\begin{aligned}
\left(\Phi^{-1} \circ F\right)(M+s) & =\left(\Phi^{-1} \circ F\right)(M+s)-\left(\Phi^{-1} \circ F\right)(M) \\
& \leq s\left(\Phi^{-1} \circ F\right)^{\prime}(M+) \\
& =s \sqrt{2 \pi} F^{\prime}(M+), \quad s \in \mathbb{R} .
\end{aligned}
$$

Now we give a lower bound for $F^{\prime}(M+)$ in terms of the standard deviation of $f(Z)$. 
Claim. We have the following:

$$
F^{\prime}(M+) \geq \frac{1}{32 \mathbb{E}(f(Z)-M)_{+}} .
$$

PROOF. Fix $\delta>0$ (that will be chosen appropriately later). Using the logconcavity of $F$, we may write

$$
\begin{aligned}
\delta \frac{F^{\prime}(M+)}{F(M)} & \geq \log F(M+\delta)-\log F(M) \\
& =\log (1+2 \mathbb{P}(M<f(Z) \leq M+\delta)) \\
& \geq \mathbb{P}(M<f(Z) \leq M+\delta) \\
& =\frac{1}{2}-\mathbb{P}(f(Z)>M+\delta),
\end{aligned}
$$

where we have used the elementary inequality $\log (1+u) \geq u / 2$ for all $0<u \leq 1$. Now we apply Markov's inequality to get

$$
\mathbb{P}(f(Z)>M+\delta) \leq \frac{\mathbb{E}(f(Z)-M)_{+}}{\delta} .
$$

Combing the above, we conclude that

$$
F^{\prime}(M+) \geq \frac{1}{2 \delta}\left(\frac{1}{2}-\frac{\mathbb{E}(f(Z)-M)_{+}}{\delta}\right) .
$$

The choice $\delta=4 \mathbb{E}(f(Z)-M)_{+}$yields the assertion of the claim.

Going back to (2.1), we readily see that $\left[\right.$ for $\left.s=-t \mathbb{E}(f(Z)-M)_{+}\right]$:

$$
\Phi^{-1}\left[\mathbb{P}\left(f(Z)-M \leq-t \mathbb{E}(f(Z)-M)_{+}\right)\right] \leq-t \frac{\sqrt{2 \pi}}{32},
$$

as required.

Let us note that one can prove a similar inequality for the $n$-dimensional exponential measure but for 1-unconditional functions $f$, that is, functions which satisfy $f\left(x_{1}, \ldots, x_{n}\right)=f\left(\left|x_{1}\right|, \ldots,\left|x_{n}\right|\right)$ for all $x=\left(x_{1}, \ldots, x_{n}\right) \in \mathbb{R}^{n}$.

We fix $W$ for an $n$-dimensional exponential random vector, that is, $W=$ $\left(\xi_{1}, \ldots, \xi_{n}\right)$, where $\left(\xi_{i}\right)_{i=1}^{n}$ are independent identically distributed according to the measure $v_{1}$ with density function $d v_{1}(x)=\frac{1}{2} e^{-|x|} d x$. Note that if $g_{1}, g_{2}$ are i.i.d. standard normals and $\xi$ is independent exponential random variable then $|\xi|$ and $\frac{g_{1}^{2}+g_{2}^{2}}{2}$ have the same distribution (follows easily by checking the moment generating functions). Based on this remark, we have the following consequence of Theorem 2.1: 
THEOREM 2.2. Let $f$ be an 1-unconditional and convex function on $\mathbb{R}^{n}$. If $W$ is an exponential random vector on $\mathbb{R}^{n}$, then one has

$$
\mathbb{P}\left(f(W)-M<-t \mathbb{E}(f(W)-M)_{+}\right) \leq 1-\Phi(c t) \leq \exp \left(-c^{\prime} t^{2}\right),
$$

for all $t>0$.

Proof. Consider the function $F: \mathbb{R}^{2 n} \rightarrow \mathbb{R}$ defined as

$$
F\left(x_{1}, \ldots, x_{n}, y_{1}, \ldots, y_{n}\right):=f\left(\frac{x_{1}^{2}+y_{1}^{2}}{2}, \ldots, \frac{x_{n}^{2}+y_{n}^{2}}{2}\right) \text {. }
$$

Since $f$ is convex and 1-unconditional, it follows that $f$ is convex and coordinatewise nondecreasing ${ }^{3}$ in the octant $\mathbb{R}_{+}^{n}=\left\{z=\left(z_{1}, \ldots, z_{n}\right): z_{i} \geq 0\right\}$. Hence $F$ is convex on $\mathbb{R}^{2 n}$. Therefore, a direct application of Theorem 2.1 yields

$$
\mathbb{P}\left(f(\tilde{W})-M<-t \mathbb{E}(f(\tilde{W})-M)_{+}\right) \leq \Phi(-c t),
$$

for all $t>0$, where $\tilde{W}=\left(\left|\xi_{1}\right|, \ldots,\left|\xi_{n}\right|\right)$ and $\xi_{i}$ are i.i.d. exponential random variables. The fact that $f\left(x_{1}, \ldots, x_{n}\right)=f\left(\left|x_{1}\right|, \ldots,\left|x_{n}\right|\right)$ completes the proof.

REMARK 2.3. In the above argument, it is clear that we may also consider longer sums of the form $g_{1}^{2}+\cdots+g_{k}^{2}$. That is, if $f: \mathbb{R}_{+}^{n} \rightarrow \mathbb{R}$ is a coordinatewise nondecreasing and convex function, then

$$
\mathbb{P}\left(f(\chi)<M-t \mathbb{E}(f(\chi)-M)_{+}\right) \leq \Phi(-t / 2),
$$

for all $t>0$, where $\chi \sim \chi^{2}(k)$ is a chi squared random variable with $k$ degrees of freedom.

We conclude this section with some remarks on the main result.

REMARKS 2.4. 1. The advantage of this one-sided concentration inequality is that it can be applied for the wide class of convex functions which are not necessarily (globally) Lipschitz or which are not even in $L_{2}\left(\gamma_{n}\right)$; for example, the function $f(t)=\exp \left(-t+t^{2} / 2\right)$ is (logarithmically) convex, belongs to $L_{1}\left(\gamma_{1}\right)$ but $f \notin L_{2}\left(\gamma_{1}\right)$. Moreover, a careful inspection of the argument shows that it is enough to have $f \in L_{1, \infty}\left(\gamma_{n}\right)$ (see, e.g., [10] for the definition of the weak $L_{p}$ space) and the conclusion still holds:

$$
\mathbb{P}\left(f(Z)<M-t\left\|(f-M)_{+}\right\|_{1, \infty}\right) \leq \Phi(-c t), \quad t>0,
$$

where $c>0$ is an absolute constant. ${ }^{4}$

\footnotetext{
${ }^{3}$ A real valued function $H$ defined on $U \subseteq \mathbb{R}^{k}$ is said to be coordinatewise nondecreasing if it is nondecreasing in each variable while keeping all the other variables fixed at any value.

${ }^{4}$ Here and everywhere else, $C$ and $c, c_{1}, \ldots$ stand for absolute constants whose values may change from line to line. We write $c(p)$ if the constant depends only on $p$.
} 
2. Assuming that $\mathbb{P}(f \leq M)=1 / 2$, then (2.1) shows that the variable $f(Z)$ stochastically dominates the normal random variable $\zeta:=M+a \cdot g$, where $g$ is a standard normal variable and $1 / a:=(2 \pi)^{1 / 2} F^{\prime}(M+)>0$, that is,

$$
\mathbb{P}(f(Z) \leq s) \leq \mathbb{P}(\zeta \leq s),
$$

for all $s \in \mathbb{R}$. Hence one gets $\mathbb{E} f(Z) \geq \mathbb{E} \zeta=M$. If $\mathbb{P}(f \leq M)>1 / 2$, then inf $f=$ $M$ and the latter is again true. This result is due to Kwapien [17]. In fact, our proof steps on the same starting line as in [17].

3. Taking into account the fact that $\mathbb{E}(f(Z)-M)_{+} \leq \mathbb{E}|f(Z)-M| \leq$ $\sqrt{\operatorname{Var} f(Z)}$ and

$$
1-\Phi(u)=\Phi(-u) \leq \frac{1}{2} e^{-u^{2} / 2}
$$

for all $u>0$ (for a proof see [19], Lemma 1) we immediately get

$$
\mathbb{P}(f(Z)-M<-t \sqrt{\operatorname{Var} f(Z)}) \leq \Phi\left(-t \frac{\sqrt{2 \pi}}{32}\right) \leq \frac{1}{2} \exp \left(-\frac{\pi}{1024} t^{2}\right),
$$

for all $t>0$, which is the announced estimate (1.5) provided that $f \in L_{2}\left(\gamma_{n}\right)$.

Furthermore, using the fact $M \geq \mathbb{E} f(Z)-\sqrt{\operatorname{Var} f(Z)}$ once more, we may conclude the following "Central Limit type" normalization in Theorem 2.1: For any convex function $f$ on $\mathbb{R}^{n}$ with $f \in L_{2}\left(\gamma_{n}\right)$, one has the following distributional inequality:

$$
\begin{aligned}
\mathbb{P}(f(Z)-\mathbb{E} f(Z) & <-t \sqrt{\operatorname{Var} f(Z)}) \\
& \leq \frac{1}{2} \exp \left(-\frac{\pi}{1024}(t-1)^{2}\right) \\
& <e^{-t^{2} / 1000}
\end{aligned}
$$

for all $t>1$.

4. Let us note that in all the above statements, one can derive the reverse distributional inequality for concave functions. Namely, if $f$ is a concave function on $\mathbb{R}^{n}$ with $f \in L_{1}\left(\gamma_{n}\right)$, then

$$
\mathbb{P}\left(f(Z)-M>t \mathbb{E}(M-f(Z))_{+}\right) \leq \Phi(-c t),
$$

for all $t>0$, where $M$ is a median for $f(Z)$.

5. We should stress the fact that in the statement of Theorem 2.1 we refer to convex functions in $L_{1}$. Thus it is pointless to ask about a similar upper estimate other than the $L_{1}$-estimate. However, in various significant applications the functions under consideration are norms or more generally Lipschitz functions, which are known to belong in $L_{\psi_{2}}\left(\gamma_{n}\right)$. In fact, $\|f-M\|_{\psi_{2}} \leq C \operatorname{Lip}(f)$ [where the $L_{\psi_{2}}$ norm stands for the Orlicz norm with Young function $\left.\psi_{2}(t)=e^{t^{2}}-1, t \geq 0\right]$. However, there are many examples of norms $f$ for which $\operatorname{Var}[f(Z)] \ll \operatorname{Lip}(f)^{2}$. 
Therefore, it is natural to ask if there is one-sided concentration estimate (in the large deviation regime), which takes into account both the variance and the Lipschitz constant. A naive approach which puts these remarks together is to combine Chebyshev's inequality with the concentration estimate in terms of the Lipschitz constant:

$$
\mathbb{P}(|f(Z)-M|>t) \leq \exp \left(-\frac{1}{2} \max \left\{\log (t / \sqrt{\operatorname{Var} f(Z)}), t^{2} / L^{2}\right\}\right) .
$$

Even in the case of a norm as above this bound depends continuously on $t>0$ and seems to be the right one. Example of such a norm is the $\ell_{p}$ norm on $\mathbb{R}^{n}$ with $p=c_{0} \log n$, for sufficiently small absolute constant $c_{0}>0$ (see [30], Section 3).

6. (Nonoptimality in $\ell_{\infty}^{n}$.) Note that Theorem 2.1 for $f(x)=\|x\|_{\infty}, x \in \mathbb{R}^{n}$ only yields

$$
\mathbb{P}\left(\|Z\|_{\infty}<(1-\varepsilon) M_{\infty, n}\right) \leq \frac{1}{2} e^{-c \varepsilon^{2} \log ^{2} n},
$$

for all $0<\varepsilon<1$, where $M_{\infty, n}$ is the median of $\|Z\|_{\infty}$. This estimate is far from being the sharp one: It is known (see [32], Claim 3) that one has

$$
\exp \left(-C e^{c^{\prime} \varepsilon \log n}\right) \leq \mathbb{P}\left(\|Z\|_{\infty}<(1-\varepsilon) M_{\infty, n}\right) \leq C \exp \left(-c e^{c \varepsilon \log n}\right),
$$

for all $0<\varepsilon<1 / 2$.

7. (Optimality in $\ell_{p}^{n}, 1 \leq p<\infty$.) In [30], it is proved that for any $1 \leq p<\infty$ one has $v_{p, n}:=\operatorname{Var}\|Z\|_{p} / M_{p, n}^{2} \leq c(p) / n$, where $M_{p, n}$ is the median for $\|Z\|_{p}$ (see also [28] for an extension of this result to any finite dimensional subspace of $\left.L_{p}\right)$. On the other hand, for any norm $\|\cdot\|$ on $\mathbb{R}^{n}$ we can deduce that

$$
\mathbb{P}(\|Z\|<(1-\varepsilon) \mathbb{E}\|Z\|) \geq c \exp \left(-C \varepsilon^{2} n\right),
$$

for all $0<\varepsilon<1 / 2$. Therefore, we obtain

$$
\mathbb{P}\left(\|Z\|_{p}<(1-\varepsilon) M_{p, n}\right) \geq c^{\prime} \exp \left(-C(p) \varepsilon^{2} / v_{p, n}\right) .
$$

8. Probabilistic inequalities similar to (1.5), in the context of log-concave measures, will be presented elsewhere [29].

3. Small ball probabilities and applications. In this section, we show that the small deviation inequality proved in Theorem 2.1 leads to new reverse Hölder inequalities for negative moments and small ball probabilities. Toward this end, we exploit once more convexity properties of the Gaussian measure by utilizing the B-inequality proved by Cordero-Erausquin, Fradelizi and Maurey in [7]. The latter states that for any centrally symmetric convex body $K$ in $\mathbb{R}^{n}$ the function

$$
t \mapsto \mathbb{P}\left(\|Z\|_{K} \leq e^{t}\right), \quad Z \sim N\left(\mathbf{0}, I_{n}\right)
$$

\footnotetext{
${ }^{5}$ A subset $K$ in $\mathbb{R}^{n}$ is said to be a centrally symmetric convex body, if it is convex, compact with nonempty interior and $K=-K$.
} 
is log-concave, where $\|\cdot\|_{K}$ is the gauge of $K$. As this result is available only for norms (the fact that the symmetry assumption is essential has been shown in [25]) from now on we will work within this context. Using the aforementioned result, and building on the ideas of Latała and Oleszkiewicz from [19], Klartag and Vershynin in [15] introduced a parameter associated with any centrally symmetric convex body which governs the small ball probability for the corresponding norm. We recall the Klartag-Vershynin parameter (in the Gaussian setting) from [15]: For any centrally symmetric convex body $A$ in $\mathbb{R}^{n}$, we define

$$
d(A):=\min \left\{n,-\log \gamma_{n}\left(\frac{M}{2} A\right)\right\},
$$

where $M$ is the median of $\|Z\|, Z \sim N\left(\mathbf{0}, I_{n}\right)$. Their result reads as follows.

THEOREM 3.1 (Klartag-Vershynin). Let A be a centrally symmetric convex body in $\mathbb{R}^{n}$. Then one has

$$
\mathbb{P}\left(\|Z\|_{A} \leq \varepsilon M\right) \leq \frac{1}{2} \varepsilon^{c d(A)}, \quad 0<\varepsilon<1 / 2,
$$

where $M$ is the median of $\|Z\|_{A}$ and $Z$ is an $n$-dimensional standard Gaussian vector.

In general, it is quite hard to estimate the quantity $d(A)$ and the known lower bounds are in general suboptimal (see Remark 3.6). The small deviation inequality from Theorem 2.1 provides a variance-sensitive lower bound for the quantity $d(A)$. For this end, we associate with any centrally symmetric convex body $A$ in $\mathbb{R}^{n}$ the following parameter:

$$
\beta(A):=\frac{\operatorname{Var}\|Z\|_{A}}{M^{2}}, \quad Z \sim N\left(\mathbf{0}, I_{n}\right),
$$

where $M$ is the median of $\|Z\|_{A}$. With this notation, we have the following.

Proposition 3.2. Let $A$ be a centrally symmetric convex body in $\mathbb{R}^{n}$. Then one has the one-sided concentration estimate

$$
\mathbb{P}\left(\|Z\|_{A} \leq(1-\varepsilon) M\right) \leq \frac{1}{2} \exp \left(-c \varepsilon^{2} / \beta(A)\right), \quad 0<\varepsilon<1,
$$

where $M$ is the median of $\|Z\|_{A}$ and $Z$ is an $n$-dimensional standard Gaussian random vector. In particular,

$$
d(A) \geq c_{1} / \beta(A),
$$

therefore, we have the following small ball probability estimate:

$$
\mathbb{P}\left(\|Z\|_{A} \leq \varepsilon M\right) \leq \frac{1}{2} \varepsilon^{c / \beta(A)}
$$

for all $\varepsilon \in(0,1 / 2)$. 
Proof. We apply Theorem 2.1 for $t=\varepsilon / \sqrt{\beta(A)}$ to get the first estimate. The bound $d(A) \geq c / \beta(A)$ follows by the definition of $d$ by plugging $\varepsilon=1 / 2$ in (3.2). Now the probabilistic estimate (3.4) follows from Theorem 3.1 and the obtained lower bound on $d(A)$.

It is known that the small ball probability (3.4) can be easily translated to a small ball probability for Gaussian processes (see, e.g., [20], Theorem 7.1), thus one has the following formulation.

THEOREM 3.3. Let $\left(G_{t}\right)_{t \in T}$ be a centered Gaussian process indexed by a countable set $T$ such that $\sup _{t \in T}\left|G_{t}\right|<\infty$ almost surely. Then, for any $\varepsilon \in$ $(0,1 / 2)$ we have

$$
\mathbb{P}\left(\sup _{t \in T}\left|G_{t}\right| \leq \varepsilon M\right) \leq \frac{1}{2} \varepsilon^{c M^{2} / v^{2}},
$$

where $M=\operatorname{med}\left(\sup _{t \in T}\left|G_{t}\right|\right)$ and $v^{2}=\operatorname{Var}\left(\sup _{t \in T}\left|G_{t}\right|\right)$.

The proof of the above theorem follows the same lines as in [19], Theorem 4, with the obvious adaptations, thus it is omitted.

In view of Theorem 2.2, one can derive small ball estimates for 1-unconditional norms with respect to the exponential measure $v_{1}^{n}$. This is promised by a result of Cordero-Erausquin, Fradelizi and Maurey, also proved in [7], that any 1unconditional log-concave measure $\mu$ and 1-unconditional convex body $K$ in $\mathbb{R}^{n}$ has the $B$-property, that is, $t \mapsto \mu\left(e^{t} K\right)$ is log-concave (recently it was proved in [9] that the B-property is satisfied by the $v_{1}^{n}$ and any centrally symmetric convex body). Although the proof is the same as in the Gaussian context, we sketch it for reader's convenience.

Proposition 3.4. Let $K$ be an 1-unconditional convex body in $\mathbb{R}^{n}$. If $W$ is a random vector distributed according to the $n$-dimensional exponential measure $v_{1}^{n}$, then one has

$$
\mathbb{P}\left(\|W\|_{K} \leq \varepsilon m\right) \leq \frac{1}{2} \varepsilon^{c / \beta}, \quad \varepsilon \in(0,1 / 2),
$$

where $m$ is the median of $\|W\|_{K}$ and $\beta=\operatorname{Var}\|W\|_{K} / m^{2}$.

PROOF. Applying Theorem 2.2 for $x \mapsto\|x\|_{K}$, we obtain

$$
v_{1}^{n}\left(\left\{x:\|x\|_{K} \leq m / 2\right\}\right)=v_{1}^{n}\left(\frac{m}{2} K\right) \leq \frac{1}{2} e^{-c / \beta} .
$$

On the other hand, since $t \mapsto v_{1}^{n}\left(e^{t} K\right)$ is log-concave, we may argue as follows: given $\varepsilon \in(0,1 / 2)$ we choose $\lambda \in(0,1)$ such that $1 / 2=\varepsilon^{1-\lambda}$, that is, $1-\lambda=$ 
$\frac{\log 2}{\log (1 / \varepsilon)}$. The log-concavity implies

$$
v_{1}^{n}\left(\frac{m}{2} K\right) \geq\left[v_{1}^{n}(\varepsilon m K)\right]^{1-\lambda}\left[v_{1}^{n}(m K)\right]^{\lambda} \quad \Longrightarrow \quad\left[2 v_{1}^{n}(\varepsilon m K)\right]^{1-\lambda} \leq 2 v_{1}^{n}\left(\frac{m}{2} K\right) .
$$

Plug (3.5) in the latter and we get the assertion.

Now we turn in proving reverse Hölder inequalities for negative moments of norms by using the small deviation (3.2) and the small ball probability (3.4):

COROLlaRY 3.5. Let $K$ be a centrally symmetric convex body in $\mathbb{R}^{n}$. Then one has

$$
\mathbb{E}\|Z\|_{K}\left(\mathbb{E}\|Z\|_{K}^{-q}\right)^{1 / q} \leq \exp (C \sqrt{\beta}+C q \beta),
$$

for all $0<q<c / \beta(K)$ where $C, c>0$ are absolute constants and $Z$ is an $n$ dimensional standard Gaussian vector.

ProOF. We know that

$$
\mathbb{P}\left(\|Z\|_{K} \leq \varepsilon M\right) \leq \frac{1}{2} \varepsilon^{c_{1} / \beta}, \quad \mathbb{P}\left(\|Z\|_{K} \leq(1-\varepsilon) M\right) \leq \frac{1}{2} e^{-c_{2} \varepsilon^{2} / \beta},
$$

for all $\varepsilon \in(0,1 / 2)$, where $M$ is the median for $\|Z\|_{K}$ and $Z \sim N\left(\mathbf{0}, I_{n}\right)$. Therefore, we may write

$$
\begin{aligned}
\mathbb{E}\|Z\|_{K}^{-q} & =M^{-q} \int_{0}^{\infty} \mathbb{P}\left(\|Z\|_{K} \leq t M\right) \frac{q}{t^{q+1}} d t \\
& \leq M^{-q}\left(\frac{q}{2} \int_{0}^{1 / 2} \varepsilon^{\frac{c_{1}}{\beta}-q-1} d \varepsilon+\int_{1 / 2}^{1} \frac{q}{t^{q+1}} P\left(\|Z\|_{K} \leq t M\right) d t+1\right) \\
& \leq M^{-q}\left(\left(\frac{1}{2}\right)^{\frac{c_{1}}{\beta}-q} \frac{q \beta}{c_{1}-q \beta}+q \int_{0}^{1 / 2} \frac{1}{(1-\varepsilon)^{q+1}} e^{-c_{2} \varepsilon^{2} / \beta} d \varepsilon+1\right) \\
& \leq M^{-q}\left(1+c_{3} q \beta+q \int_{0}^{1 / 2} \exp \left(2(q+1) \varepsilon-c_{2} \varepsilon^{2} / \beta\right) d \varepsilon\right),
\end{aligned}
$$

for all $0<q<c_{4} / \beta$, where we have also used the elementary inequality $1-u \geq$ $e^{-2 u}$ for $0 \leq u \leq 1 / 2$. It is easy to check that the last integral can be bounded as

$$
\int_{0}^{1 / 2} \exp \left(2(q+1) \varepsilon-c_{2} \varepsilon^{2} / \beta\right) d \varepsilon \leq c_{5} \sqrt{\beta} \exp \left(c_{5} q^{2} \beta\right),
$$

for all $0<q \leq c_{6} / \beta$. The result follows.

REMARK 3.6. Klartag and Vershynin in [15] observed that the concentration of measure inequality (1.4) implies that $d(A) \geq \operatorname{ck}(A)$ where $k(A)$ is given by

$$
k(A):=\mathbb{E}\|Z\|_{A}^{2} / b(A)^{2}, \quad b(A)=\max _{\theta \in S^{n-1}}\|\theta\|_{A} .
$$


The quantity $k(A)$ is introduced by V. Milman in [23] and it is usually referred to as the critical dimension of the body $A$. We refer to [24] for further information on this quantity. Although the quantity $k(A)$ is easy to be computed, there are several cases in which bounding $d(A)$ by $k(A)$ gives suboptimal results. Using (1.3) and the fact $\mathbb{E}\|Z\| \leq c M$, it is clear that $\frac{1}{\beta(A)} \geq c^{\prime} k(A)$, thus Proposition 3.2 provides better bounds for the quantity $d(A)$. We illustrate this in the following example: Consider as convex body $A$ the unit ball of some $n$-dimensional subspace of $L_{p}, 2<p<\infty$. It is proven in [28] that there exists a linear image $\tilde{A}$ of $A$ with $\beta(\tilde{A}) \leq C(p) / n$ while $k(\tilde{A})$ can be of the order $n^{2 / p}$ (up to constants depending only on $p$ ). In this case, the bounds given by Proposition 3.2 are sharp (up to constants depending only on $p$ ).

The inequalities presented in the paper can be used to obtain refinements of several classical results in asymptotic geometric analysis such as the random version of Dvoretzky's theorem [23]. These applications will appear elsewhere [29], [27]. We close this section by mentioning one interesting application of the results to the Johnson-Linderstrauss flattening lemma.

The J-L lemma from [13] (see also [14]) asserts that: if $\varepsilon \in(0,1)$ and $x_{1}, \ldots, x_{N} \in \ell_{2}$, then there exists a linear mapping (which can be chosen to be an orthogonal projection) $P: \ell_{2} \rightarrow F$, where $F$ is a subspace of $\ell_{2}$ with $\operatorname{dim} F \leq c \varepsilon^{-2} \log N$ such that

$$
(1-\varepsilon)\left\|x_{i}-x_{j}\right\|_{2} \leq\left\|P x_{i}-P x_{j}\right\|_{2} \leq(1+\varepsilon)\left\|x_{i}-x_{j}\right\|_{2},
$$

for all $i, j=1, \ldots, N$.

This dimension reduction principle has found various applications in mathematics and computer science, in addition to the original application in [13] for the Lipschitz extension problem. We refer the interested reader to $[11,16,35]$ and the references therein for a partial list of its many applications.

The J-L lemma we are interested in applies for arbitrary target spaces, as was formulated in [31]. Below we suggest a refined one-sided version of the latter.

Proposition 3.7. Let $X=\left(\mathbb{R}^{n},\|\cdot\|\right)$ be a normed space and let $T \subseteq \ell_{2}^{N}$ be a finite set with $T=\left\{u_{1}, \ldots, u_{N}\right\}$. The following hold:

(i) Let $\delta \in(0,1)$ and assume that $\log |T| \leq c \delta^{2} / \beta(X)$. Then the random Gaussian matrix $G=\left(g_{i j}\right)_{i, j=1}^{n, N}$ satisfies

$$
\left\|G u_{i}-G u_{j}\right\| \geq(1-\delta) \cdot \mathbb{E}\|Z\| \cdot\left\|u_{i}-u_{j}\right\|_{2}
$$

for all $i, j,=1, \ldots, N$, where $Z \sim N\left(\mathbf{0}, I_{n}\right)$, with probability greater than 1 $c e^{-c \delta^{2} / \beta(X)}$. 
(ii) Let $\varepsilon \in(0,1 / 2)$ and assume that $\log |T| \leq c \log (1 / \varepsilon) / \beta(X)$. Then the random Gaussian matrix $G=\left(g_{i j}\right)_{i, j=1}^{n, N}$ satisfies

$$
\left\|G u_{i}-G u_{j}\right\| \geq c \varepsilon \cdot \mathbb{E}\|Z\| \cdot\left\|u_{i}-u_{j}\right\|_{2},
$$

for all $i, j,=1, \ldots, N$, where $Z \sim N\left(\mathbf{0}, I_{n}\right)$, with probability greater than $1-$ $c \varepsilon^{c / \beta(X)}$.

Proof. Consider $Z_{1}, \ldots, Z_{N}$ i.i.d. standard Gaussian vectors on $\mathbb{R}^{n}$ and define the random matrix $G=\left[Z_{1}, \ldots, Z_{N}\right]$. Fix $\theta \in S^{N-1}$ and applying Theorem 2.1 (as was formulated further in Remark 2.4.3) we get

$$
\mathbb{P}(\|G \theta\|<(1-t) \mathbb{E}\|Z\|)=\mathbb{P}\left(\left\|Z_{1}\right\|<\mathbb{E}\|Z\|-t \mathbb{E}\|Z\|\right) \leq C \exp \left(-c t^{2} / \beta\right),
$$

for all $t \in(0,1)$. If $T=\left\{u_{1}, \ldots, u_{N}\right\}$, consider the set of points

$$
\Theta:=\left\{\frac{u_{i}-u_{j}}{\left\|u_{i}-u_{j}\right\|_{2}}: 1 \leq i<j \leq N\right\}
$$

on the Euclidean sphere $S^{N-1}$. Then by the union bound we get

$$
\mathbb{P}(\exists \theta \in \Theta:\|G \theta\|<(1-\delta) \mathbb{E}\|Z\|)<C_{1} N^{2} \exp \left(-c_{1} \delta^{2} / \beta\right) \leq C_{2} \exp \left(-c_{2} \delta^{2} / \beta\right),
$$

as long as $\log N \leq c \delta^{2} / \beta(X)$. The assertion follows.

The same reasoning as above, but using (3.4) instead, yields (ii).

Acknowledgments. The authors are grateful to Ramon van Handel for useful discussions and to Mark Rudelson for important remarks. They would also like to thank the anonymous referee whose valuable comments helped to improve the presentation of this paper.

\section{REFERENCES}

[1] BogacheV, V. I. (1998). Gaussian Measures. Mathematical Surveys and Monographs 62. Amer. Math. Soc., Providence, RI. MR1642391

[2] Borell, C. (1975). The Brunn-Minkowski inequality in Gauss space. Invent. Math. 30 207216. MR0399402

[3] Borell, C. (2003). The Ehrhard inequality. C. R. Math. Acad. Sci. Paris $337663-666$. MR2030108

[4] Boucheron, S., Lugosi, G. and Massart, P. (2013). Concentration Inequalities: A Nonasymptotic Theory of Independence. Oxford Univ. Press, Oxford. MR3185193

[5] Chatterjee, S. (2014). Superconcentration and Related Topics. Springer, Cham. MR3157205

[6] CHEN, L. H. Y. (1982). An inequality for the multivariate normal distribution. J. Multivariate Anal. 12 306-315. MR0661566

[7] Cordero-Erausquin, D., Fradelizi, M. and Maurey, B. (2004). The (B) conjecture for the Gaussian measure of dilates of symmetric convex sets and related problems. J. Funct. Anal. 214 410-427. MR2083308 
[8] Ehrhard, A. (1983). Symétrisation dans l'espace de Gauss. Math. Scand. 53 281-301. MR0745081

[9] Eskenazis, A., NAYAR, P. and TKocz, T. (2016). Gaussian mixtures: Entropy and geometric inequalities. Preprint. Available at https://arxiv.org/abs/1611.04921.

[10] Grafakos, L. (2004). Classical and Modern Fourier Analysis. Pearson Education, Upper Saddle River, NJ. MR2449250

[11] INDYK, P. (2001). Algorithmic applications of low-distortion geometric embeddings. In 42nd IEEE Symposium on Foundations of Computer Science (Las Vegas, NV, 2001) 10-33. IEEE Computer Soc., Los Alamitos, CA. MR1948692

[12] IVAnisvili, P. and VolberG, A. (2015). Bellman partial differential equation and the hill property for classical isoperimetric problems. Preprint. Available at https://arxiv.org/abs/ 1506.03409 .

[13] Johnson, W. B. and Lindenstrauss, J. (1984). Extensions of Lipschitz mappings into a Hilbert space. In Conference in Modern Analysis and Probability (New Haven, Conn., 1982). 189-206. Amer. Math. Soc., Providence, RI. MR0737400

[14] Johnson, W. B. and NAOR, A. (2010). The Johnson-Lindenstrauss lemma almost characterizes Hilbert space, but not quite. Discrete Comput. Geom. 43 542-553. MR2587836

[15] Klartag, B. and Vershynin, R. (2007). Small ball probability and Dvoretzky's theorem. Israel J. Math. 157 193-207. MR2342445

[16] Kushilevitz, E., Ostrovsky, R. and Rabani, Y. (2000). Efficient search for approximate nearest neighbor in high dimensional spaces. SIAM J. Comput. 30 457-474. MR1769366

[17] KWAPIEŃ, S. (1994). A remark on the median and the expectation of convex functions of Gaussian vectors. In Probability in Banach Spaces, 9 (Sandjberg, 1993). Progress in Probability 35 271-272. Birkhäuser, Boston, MA. MR1308523

[18] LataŁA, R. (1996). A note on the Ehrhard inequality. Studia Math. 118 169-174. MR1389763

[19] LatąA, R. and OleszKiewicz, K. (2005). Small ball probability estimates in terms of widths. Studia Math. 169 305-314. MR2140804

[20] LedouX, M. (2001). The Concentration of Measure Phenomenon. Mathematical Surveys and Monographs 89. Amer. Math. Soc., Providence, RI. MR1849347

[21] Ledoux, M. and TAlagrand, M. (1991). Probability in Banach Spaces. Isoperimetry and Processes. Springer, Berlin. MR1102015

[22] Litvak, A. E., Milman, V. D. and Schechtman, G. (1998). Averages of norms and quasinorms. Math. Ann. 312 95-124. MR1645952

[23] Milman, V. D. (1971). New proof of the theorem of A. Dvoretzky on sections of convex bodies (in Russian). Funkcional. Anal. i Prilozen. 5 28-37. MR0293374

[24] Milman, V. D. and Schechtman, G. (1986). Asymptotic Theory of Finite-Dimensional Normed Spaces. Lecture Notes in Math. 1200. Springer, Berlin. MR0856576

[25] Nayar, P. and Tкосz, T. (2013). A note on a Brunn-Minkowski inequality for the Gaussian measure. Proc. Amer. Math. Soc. 141 4027-4030. MR3091793

[26] Neeman, J. and Paouris, G. (2016). An interpolation proof of Ehrhard's inequality. Preprint. Available at https://arxiv.org/abs/1605.07233.

[27] Paouris, G., Pivovarov, P. and Valettas, P. (2017). On a quantitative reversal of Alexandrov's inequality. Trans. Amer. Math. Soc. To appear. Available at https://arxiv.org/abs/ 1702.05762 .

[28] Paouris, G. and Valettas, P. (2015). On Dvoretzky's theorem for subspaces of $L_{p}$. Preprint. Available at http://arxiv.org/abs/1510.07289.

[29] Paouris, G. and Valettas, P. (2017). Variance estimates and almost Euclidean structure. Preprint. Available at https://arxiv.org/abs/1703.10244.

[30] Paouris, G., Valettas, P. and ZinN, J. (2017). Random version of Dvoretzky's theorem in $\ell_{p}^{n}$. Stochastic Process. Appl. 127 3187-3227. MR3692312 
[31] Schechtman, G. (2006). Two observations regarding embedding subsets of Euclidean spaces in normed spaces. Adv. Math. 200 125-135. MR2199631

[32] Schechtman, G. (2007). The random version of Dvoretzky's theorem in $\ell_{\infty}^{n}$. In Geometric Aspects of Functional Analysis. Lecture Notes in Math. 1910 265-270. Springer, Berlin. MR2349612

[33] SudAKov, V. N. and TSIREL'son, B. S. (1974). Extremal properties of half-spaces for spherically invariant measures (in Russian). Zap. Naučn. Sem. Leningrad. Otdel. Mat. Inst. Steklov. (LOMI) 41 14-24. MR0365680

[34] VAn Handel, R. (2017). The Borell-Ehrhard game. Probab. Theory Related Fields To appear. Available at DOI:10.1007/s00440-017-0762-4.

[35] Vempala, S. S. (2004). The Random Projection Method. DIMACS Series in Discrete Mathematics and Theoretical Computer Science 65. Amer. Math. Soc., Providence, RI. MR2073630

DEPARTMENT OF MATHEMATICS

TEXAS A\&M UNIVERSITY

MAILSTOP 3368

College Station, Texas 77843-3368

USA

E-MAIL: grigorios.paouris@gmail.com

URL: http://www.math.tamu.edu/ grigoris/
MATHEMATICS DEPARTMENT

UNIVERSITY OF MISSOURI

COLUMBIA, MisSOURI 65211

USA

E-MAIL: valettasp@missouri.edu

URL: http://faculty.missouri.edu/ valettasp/ 